\title{
The diagnostic accuracy of multi-organ point-of-care ultrasonography for life-threatening conditions: The case of cancer patients who visit the emergency department: retrospective observational study
}

\section{Yun Ang Choi}

Seoul National University Hospital, Seoul National University Hospital https://orcid.org/0000-0002-2051-5963

Min Sung Lee ( $\square$ lylm85@gmail.com )

Seoul National University Hospital, Seoul National University Hospital https://orcid.org/0000-0001-9247-2432

\section{Tae Kwon Kim}

Keimyung University Dongsan Hospital: Keimyung University Dongsan Medical Center, Keimyung University Dongsan Hospital:

Keimyung University Dongsan Medical Center

Jae Yun Jung

Seoul National University Hospital, Seoul National University Hospital

\section{Ki Hong Kim}

Seoul National University Hospital, Seoul National University Hospital

\section{Joong Wan Park}

Seoul National University Hospital, Seoul National University Hospital

Jayoun Kim

Seoul National University Hospital, Seoul National University Hospital

\section{Yong Hee Lee}

Seoul National University Hospital, Seoul National University Hospital

\section{Stephen Gyung Won Lee}

Seoul Metropolitan Boramae Hospital: Seoul National University Seoul Metropolitan Government Boramae Medical Center, Seoul Metropolitan Boramae Hospital: Seoul National University Seoul Metropolitan Government Boramae Medical Center

\section{Original research}

Keywords: Point-of-care ultrasonography, Emergency department, Cancer, Critical illness, Diagnosis

Posted Date: May 17th, 2021

DOI: https://doi.org/10.21203/rs.3.rs-473861/v1

License: @ (1) This work is licensed under a Creative Commons Attribution 4.0 International License. Read Full License 


\section{Abstract}

Background The number of cancer patients visiting the emergency department (ED) is increasing globally, and this has highlighted the importance of diagnosing and coping with related life-threatening complications early. There may be efficacy in employing multiorgan point-of-care ultrasonography (M-PoCUS) for this purpose; however, there has been no study on the usefulness of this diagnostic tool for cancer patients only. The aim of this study was to evaluate the diagnostic accuracy of M-PoCUS for a lifethreatening condition in cancer patients who visited the ED.

Methods We conducted a retrospective observational study in one tertiary university hospital ED in Seoul, Republic of Korea. We selected three emergency medicine specialists to perform a protocolized M-PoCUS evaluation of cancer patients according to the requests of emergency physicians since the SARS-CoV-2 pandemic began. We enrolled 94 cancer patients in the study. The M-PoCUS diagnosis was then compared with an audit diagnosis. The primary outcomes measured were the sensitivity, specificity, positive predictive value, and negative predictive value of M-PoCUS in the above-described diagnosis.

Results The M-PoCUS showed a sensitivity of $83 \%$ (95\% Cl, 61-95), specificity of $96 \%$ (95\% Cl, 88-99), positive predictive value of $86 \%$ (95\% Cl, 65-97), and negative predictive value of $94 \%$ (95\% Cl, 85-97). M-PoCUS produced 4 false negative (4.3\%) pulmonary thromboembolism diagnoses and 3 false positive (3.2\%) pleural effusion diagnoses.

Conclusions M-PoCUS was useful in ruling out a life-threatening condition among the cancer patients. However, pulmonary thromboembolism was hard to distinguish using M-PoCUS due to pre-existing diseases scattered in cancer patients' thorax.

\section{Background}

Point-of-care ultrasonography (PoCUS) has become a very important diagnostic tool for emergency physicians in terms of its radiation-free, quickness, and accuracy [1-3]. In fact, ultrasound in general is vigorously used in various emergency department (ED) settings, not only for diagnostic modality, but also for procedural success $[4,5]$. It is well known that lung ultrasound (LUS) has higher sensitivity than x-rays for pneumonia, pneumothorax, pulmonary edema, and pleural effusion [6-13]. In pulmonary thromboembolism, the combination of focused transthoracic echocardiography (TTE), LUS and lower extremity vein ultrasound (LEVUS) showed higher sensitivity $[14,15]$. Focused TTE with LUS has been valuable in discriminating cardiogenic from non-cardiogenic dyspnea [16-18]. Therefore, multi-organ point-of-care ultrasonography (M-PoCUS), which incorporates these methods, showed high diagnostic accuracy in the ED for undifferentiated hypotension and respiration symptoms and reduced time to diagnosis in the ED [19-22]. However, for most PoCUS studies, cancer patients accounted for only $5-10 \%$ of their cohorts [20,21]. Thus, in our knowledge, the diagnostic accuracy and usefulness of M-PoCUS for cancer patients only in the ED is yet to be proven, prompting a more focused investigation into the efficacy of this method in this context.

\section{Importance}

Because the survival rate of cancer patients is increasing due to the advancement of modern medicine the number of cancer patients visiting the ED is also increasing [23,24]. In addition, the number of cancer patients requiring palliative care is also increasing [25-27]. Therefore, emergency physicians need to have a rapid approach to address the many complaints and complications commonly voiced and experienced by cancer patients. Regarding these complications, according to previous studies, it is known that respiratory distress, fever, and pain are the main symptoms of cancer patients visiting the ED; particularly, respiratory distress, shock, and sepsis are known to be associated with poor clinical outcome $[23,28]$. Considering the efficacy of M-PoCUS for the general population of ED, we expected that life-threatening conditions, common in cancer patients, could also be accurately diagnosed using M-PoCUS.

\section{Goals of this investigation}

The goal of the present study was to evaluate the diagnostic accuracy of M-PoCUS for a life-threatening condition in cancer patients who visited the ED. We hypothesized that M-PoCUS incorporated with initial clinical evaluation could correctly identify pre-defined lifethreatening conditions in cancer patients.

\section{Methods}

\section{Study design and setting}


This retrospective, single center observation study was conducted at the ED of the Seoul National University Hospital, Republic of Korea. This center is a tertiary level university hospital with approximately 60,000 annual adult ED visits. Of these, cancer patients accounted for an average of 15,000 (25\%) for a year, most of them are evaluated and treated at the ED for various complications related to underlying cancer. In this institution, the PoCUS program has been included in emergency medicine resident training since April 2018, and emergency medicine specialists with over 5 years of experience in emergency ultrasound have been conducting bedside teaching. Every year, thousands of PoCUS examinations are performed in our institution, including heart, lung, abdomen, deep vein, procedural ultrasound, airway ultrasound, and extended focused assessments with sonography for trauma [29]. Since the onset of the SARS-CoV-2 pandemic, we started to use M-PoCUS for heart, lung, inferior vena cava (IVC), and lower extremity vein examinations as initial modality more actively in order to evaluate life-threating conditions in cancer patients that had been visiting the ED since February 2020. M-PoCUS was available during the daytime and performed primarily at the request of duty emergency physicians in patients complaining of dyspnea, chest pain, shock, and hypoxia. In addition it was also performed when requested in patients with fever, gastrointestinal symptoms, and general weakness. All the examinations were performed by three emergency medicine specialists (YAC, TKK, MSL), each with experience of performing more than 200 focused TTE, 100 LUS, and 100 LEVUS previously. This study was approved by our institutional review board and met the criteria for exemption from informed consent. (Seoul National University IRB No. 2010-110-1165)

\section{Selection of Participants}

We retrospectively reviewed our institution's electrical medical records since February 2020 . Any cases with M-PoCUS performed at the ED were considered eligible for the current study. The inclusion criteria were as follows; active cancer patients [30] admitted to the ED during the study period, patients older than 18 years old, and patients whose M-PoCUS examinations were performed by selected emergency medicine specialist during their ED visit. Patients whose ultrasound image quality was poor due to their poor cooperation, resulting in inadequate retrospective interpretation, were excluded.

\section{Measurements}

M-PoCUS was performed with a multiprobe portable machine (Venue GE) with a 1.6-4.5 MHz phased array probe, 4.0-12.0 MHz linear probe, and 1.5-6.0 MHz convex probe. The requested emergency medicine specialist performed M-PoCUS after reviewing the patients' chief complaints and past medical histories. All examinations were performed according to a predefined M-PoCUS protocol described as follows.

Cardiac view

A cardiac 1.6-4.5 MHz phased array probe was used for examining the heart through the subcostal, parasternal long axis, and the apical four chamber and/or five chamber views. The visual estimation of the left ventricle (LV) systolic function was examined by the parasternal long axis and/or apical 4 chamber view [31,32]. The LV systolic function was qualitatively classified as hyperdynamic when LV ejection fraction (EF) was $\geq 70 \%$, normal for LV EF $50-70 \%$, mild to moderate dysfunction for LV EF $30-49 \%$, and severe dysfunction for $\mathrm{LV} E F<30 \%$ according to the American college of cardiology (ACC). Right ventricular (RV) dilatation was diagnosed in the presence of at least one of the following criteria: right/left ventricular end-diastolic diameter ratio $>0.9$ in the apical four chamber or subcostal view [33-35]. RV strain was indicated by the presence of septal dyskinesia or flattening [32]. Cardiac tamponade was diagnosed whenever pericardial effusion was associated with right atrium (RA) collapse and/or RV collapse during diastole plus a plethoric IVC [36, 37]. The IVC maximal diameter (end-expiration), minimal diameter (end-inspiration), and IVC collapsibility index (= maximal diameter - minimal diameter / maximal diameter) were measured in the subcostal view at 0.5 to $2.0 \mathrm{~cm}$ from the RA junction [38].

Lung view

A cardiac 1.6-4.5 MHz phased array probe was mainly used for the lung study; $4.0-12.0 \mathrm{MHz}$ linear probe or $1.5-6.0 \mathrm{MHz}$ convex probe were used together according to the ED ultrasound examiner's preference. The standardized eight anterior-posterolateral areas examination was used $[39,40]$. An LUS test was performed with the patients in the supine or near-to-supine position, or in the semi fowler's position if the patient was unable to sustain the supine position because of aggravating symptoms. A normal lung was defined as showing A-lines with the lung sliding on all eight areas without alveolar consolidation or pleural effusion [41]. B-pattern was defined as the presence of three or more B-lines between two ribs in a single scan, which is indicative of interstitial syndrome [39, 42]. B-pattern was classified as either focal or diffuse $[40,43]$. Consolidation of the lung was visualized as an echo poor or tissue like 
pattern, depending on the extent of aeration loss and fluid predominance [44]. Pleural abnormalities were defined as a pleural thickening, irregularities of the pleural margins, or small bands of consolidations [40,43]. Pleural effusions were categorized as simple or complex. Simple pleural effusions are anechoic, while complex pleural effusion are echogenic, with or without septations $[45,46]$. The use of terms 'small, moderate, and large' in reference to pleural effusions is common, with no consensus on these size limits [47]. Therefore, our team defined moderate pleural effusion as a maximal interpleural distance $\geq 25 \mathrm{~mm}(\geq 500 \mathrm{ml})[48,49]$. Pneumothorax was defined as the presence of lung point, an absence of lung sliding, an absence of lung pulse, an absence of B-line, an absence of consolidation, and an absence of pleural effusion [39].

Lower extremity vein view

A 4.0-12.0 MHz linear probe was used for the lower extremity vein study. We used the two-point (two zone) deep vein thrombosis (DVT) compression examination [50]. The regions of common femoral vein (CFV) and the greater saphenous vein (GSV) bifurcation, as well as the popliteal vein trifurcation region were scanned for compressibility; a positive DVT scan was recorded whenever the vein was not compressible or thrombus was identified in the venous lumen.

The M-PoCUS findings and diagnoses were reported in a predefined standardized form (see Additional file 1). For each patient, up to two concomitant sonographic diagnoses could be present, and the most crucial diagnosis that caused patients' symptoms was entered in the first column. Tumor progression was one of ten M-PoCUS diagnoses. However, this diagnosis had to be entered in the second column. This is because tumor progression can concomitantly exist with any acute illnesses in the first column, and the ultrasonographic definition of tumor progression has not been studied. All patient video clips and images were saved and transferred to a picture archiving communication system of Seoul National University Hospital.

The electronic medical records of all patients were reviewed in structured forms. In addition to the patients' demographics and other baseline characteristics, "time to ultrasound", and other time to procedure variables were gathered to evaluate the time-dependent ultrasound efficacy. Specifically, age, sex, type of cancer, reason of requested study, eastern cooperative oncology group (ECOG) performance status, time of ED arrival, time to start M-PoCUS, time to end M-PoCUS, time to perform x-ray, time to perform computed tomography (CT), time to administer antibiotic, time to fluid bolus infusion, time to administer diuretic, time to pleurocentesis, time to pericardiocentesis, M-PoCUS findings, and M-PoCUS diagnoses were abstracted from electronic medical record system.

\section{Outcomes}

The M-PoCUS diagnoses were cardiogenic pulmonary edema (CPE), bilateral alveolar interstitial syndrome (AIS), unilateral AIS, pleural effusion (moderate to large), pulmonary thromboembolism (PTE), cardiac tamponade, pneumothorax (PTX), hypovolemia (including distributive shock), tumor progression and normal or other disease. The AIS, which is a radiologic term, was included in the M-PoCUS diagnoses because it is the same ultrasonographic findings that cancer patients might also exhibit with a variety of lung diseases, such as primary lung cancer, solid metastasis, lymphangitic metastasis, infectious pneumonia, and drug-induced interstitial lung disease. Diagnoses and corresponding ultrasonographic findings combination were given (see Additional file 2). The predefined acute life-threatening conditions have consisted of CPE, pleural effusion (moderate to large), cardiac tamponade, PTE, PTX. Those conditions require urgent treatment such as diuresis, pleurocentesis, pericardiocentesis, anticoagulation, or tube thoracostomy.

The reference standard was the audit clinical diagnosis using an electronic chart review. For each patient, up to two concomitant diagnoses could be present. The main diagnosis was placed in the first column when two concomitant diagnoses were present. Tumor progression must be entered in the second column. At least one month after the patient visits the ED, two emergency medicine specialists reviewed all relevant clinical documentation of cases, including laboratory data, imaging tests except M-PoCUS examinations, consultative reports, and other data recorded during the hospital stay. The auditors were blinded to the results of the initial ultrasonography evaluation. The electronic chart review consisted of two separate steps. First, each auditor independently formulated one or two clinical diagnoses for the leading causes of patients' visits to the ED using ten diagnoses, the same as the MPoCUS diagnoses. In addition, they described a definite diagnosis corresponding to 10 categories of audit diagnosis. Secondly, the two auditors, joined by another emergency medicine specialist, held a plenary meeting and discussed cases where the two independent personal diagnoses were not concordant. When the plenary discussion did not create full consensus, the contradiction was resolved by the majority. Cases without the agreement of at least two of the three auditors were described as indeterminate cases.

\section{Analysis}


In one study, a presumed ED diagnosis using chest x-ray was able to diagnose life-threatening conditions with a sensitivity of $65 \%$ and a specificity of $78 \%$ [20]. The sensitivity and specificity of PoCUS for diagnosing life-threatening conditions in the ED were around $90 \%$, and there were only a few cancer patients enrolled [20]. Though we cannot predict the diagnostic performance of M-PoCUS for cancer patients, the sensitivity may slightly decrease when discriminating life-threatening conditions caused by similar ultrasonographic findings of primary cancer and/or metastatic cancer in the chest. We preliminarily reviewed some cancer patients who visited the ED for the same period and set the expected prevalence of life-threatening conditions as $30 \%$. When we set the expected sensitivity as $86 \%$ (a $5 \%$-lower value based on the above-mention $90 \%$ level), $90 \%$ for specificity, $5 \%$ for type I error, and $80 \%$ for power. Ninety-three subjects were calculated for the adequate sample size.

To assess the diagnostic performance of M-PoCUS for the predefined acute life-threatening conditions, sensitivity, specificity, positive predictive values (PPVs), negative predictive values (NPVs), and their $95 \%$ confidence intervals were calculated. For the secondary outcome, Cohen's kappa inter-rater coefficient was used to evaluate the agreement between nine M-PoCUS diagnoses and audit diagnoses. The reference standard was the first column of the audit diagnosis among the calculations. Cohen's kappa inter-rater coefficient was also used to evaluate the agreement of the two audits' first column diagnoses. For the descriptive analysis, continuous variables were shown by means with standard deviation (SD) or medians with interquartile range (IQR). Two-sided P-values of less than 0.05 were considered statistically significant. All the statistical analyses and sample size calculations were done using $R$ software version 4.0.4 (R Foundation for Statistical Computing, Vienna, Austria).

\section{Results}

\section{Characteristics of study subjects}

From March 1, 2020, to October 31, 2020, 1286 PoCUS cases were performed in our ED. We recorded 98 patients who were eligible for participation in the study. Among them, four patients were excluded due to low quality of their archived M-PoCUS images. Finally, 94 patients were enrolled in the study (Figure 1). Among them, 62 were men, and the mean age of the entire sample was $67.1 \pm 10.3$ years. Lung cancer was the most common type of cancer and dyspnea was the most common reason of requested study. The baseline characteristics are shown in Table 1.

After the patient arrived at the ED, the median time to M-PoCUS was 63.0 minutes (Interquartile range [IQR], 39.0-195.0). The median time to complete M-PoCUS evaluation was 10 minutes (IQR, 7-14). For chest X-ray and CT, the median time was 75.0 minutes (IQR, 46.0-111.5) and 240.5 minutes (IQR, 148.0-467.0), respectively. There was no adverse event associated with M-PoCUS. No complications were reported after interventional procedures such as pleurocentesis and pericardiocentesis were conducted. All intervention data are specified in Table 2.

\section{Main results}

Cardiac and lung views were performed in 94 (100\%) patients. A lower extremity vein view was performed only in $17(18.1 \%)$ patients. The M-PoCUS findings are given in Table 3.

The M-PoCUS and audit diagnosis are reported in Table 4. Overall concordance between the two audits was substantial (Cohen's kappa, 0.763) [51]. There was no indefinite case in the audit diagnosis. No examiners diagnosed pneumothorax through M-PoCUS, and there was no pneumothorax audit diagnosis. The main outcome of M-PoCUS for the predefined acute life-threatening conditions is shown in Table 5. M-PoCUS had a sensitivity of $83 \%$ (95\% Cl, 61-95); specificity of 96\% (95\% Cl, 88-99); PPV of $86 \%$ (95\% Cl, 6597); NPV of $94 \%(95 \% \mathrm{Cl}, 65-98)$ and accuracy of $93 \%(95 \% \mathrm{Cl}, 85-97)$ for detecting life-threatening conditions. The secondary outcome, the overall agreement of the nine diagnoses between the M-PoCUS and audit was substantial (Cohen's kappa, 0.742) [51].

We missed four patients with PTE when using M-PoCUS. Four false negative cases were diagnosed as unilateral AIS $(n=2)$, bilateral AIS $(n=1)$ and hypovolemia/distributive $(n=1)$ in M-PoCUS diagnosis. Three false positive cases were reported as pleural effusion in the M-PoCUS diagnosis but were diagnosed as hypovolemia/distributive $(n=2)$ and bilateral AIS $(n=1)$ in the audit diagnosis.

Definite diagnoses are reported in Table 6. The most common diagnoses were pneumonia $(n=29,30.9 \%)$, increased tumor burden $(n=26,27.6 \%)$, and pleural effusion $(n=15,16.0 \%)$.

\section{Discussion}


This investigation is the first study to evaluate the diagnostic accuracy of M-PoCUS for a life-threatening condition in cancer patients. In our study, M-PoCUS for cancer patients has shown slightly lower sensitivity in diagnosing life-threatening conditions than the previous study. Laursen et al [20]. examined that M-PoCUS has a sensitivity of $100 \%$ and specificity of $93.3 \%$ in diagnosing lifethreatening conditions in ED patients whose chief complaints were respiratory difficulty, desaturation, cough, or chest pain. Identified 19 patients with life-threatening conditions by M-PoCUS were as follows; 4 cases of PTE including 3 cases of DVT, 9 cases of heart failure with pulmonary edema, 1 case of pericardial effusion, 4 cases of empyema including 1 large amount pleural effusion, and 1 case of pneumonia with large amount pleural effusion. In contrast, we missed 4 cases of PTE. The current study's lower sensitivity is most likely due to the cancer patients' thorax condition. A meta-analysis showed that LUS offers sensitivity and specificity of $77 \%$ and $75 \%$, respectively, for PTE [52]. Those studies from the meta-analysis mainly detected the presence of triangular/wedge or rounded pleural-based lung lesions, as well as hypo-echogenic, homogeneous, pleural-based lung lesions. However, there are also many similar pleural-based lung lesions in cancer patients due to other pathologic lung diseases such as pneumonitis, primary cancer, or metastasis; thus, the sensitivity of PTE with LUS was reduced and not quite as effective. A diagnostic test study including LUS, TTE, and LEVUS showed sensitivity and specificity of $90 \%$ and of $86.2 \%$, respectively, for PTE [14]. However, our team performed LEVUS only in $18 \%$ of patients. This lower performance of LEVUS may have further decreased the sensitivity for PTE. A plausible explanation of lower performance of LEVUS is that examiners began to examine the lungs or heart first. When pathogenic findings were suspected in the process, they tended not to look at the lower extremity vein view. If LEVUS had been performed in the majority of patients, then the sensitivity of M-PoCUS would have been improved

The missed life-threatening conditions were 4 cases of PTE. In the audit diagnosis, they were confirmed by chest CT angiography including chest pulmonary artery and deep vein angiography in two cases, chest CT contrast without angiography in one case, and abdominal CT angiography in 1 case. Of them, only one DVT case was confirmed using chest CT angiography including chest pulmonary artery and deep vein angiography. DVT could not be identified in the remaining cases due to limited CT protocols (Chest CT without angiography, abdominal CT angiography). In the TTE finding of M-PoCUS, there were no cases of RV dilatation and IVC plethora. In the LUS findings, pleural-based lung lesions were shown as combined with other finding such as lobar consolidation or B pattern in 3 cases, and 1 case was shown as normal. As mentioned earlier, those LUS findings suggest that it is difficult to rule in PTE through only pleural-based lung lesions in cancer patients. Even if there was an alternative diagnosis in LUS and/or TTE, PTE can be present in those cancer patients. Therefore, in the case of cancer patients, if PTE is clinically suspected, we believe that it might be better to actively perform CT angiography.

The false positive cases were 3 cases of pleural effusion. For those cases, the audit diagnoses were hypovolemic or distributive for 1 case and bilateral AIS for the remaining 2 cases. Even in the presence of a moderate amount of pleural effusion, the audits determined that sepsis, pneumonia, and tumor progression were the final diagnosis for false positive cases. In light of these false positive cases, we believe that when diagnosing the pleural effusion as the main diagnosis for cancer patients, careful clinical interpretation considering previous statuses must be combined to lower false positive rates. That way, emergency physicians will be able to detect problems that are more important to their patients and prevent unnecessary pleurocentesis and consequent adverse events.

\section{Limitations of the study}

The major limitation of this study is the retrospective design. Due to the study design, the reference standard could not be predefined. For example, cardiac tamponade must be confirmed with advanced transthoracic echocardiography (TTE), which is performed and interpreted by cardiologists. However, in our ED settings, unstable patients with highly suspected cardiac tamponade screened through M-PoCUS were treated by emergency pericardiocentesis with cardiologist' interpretations of M-PoCUS instead of advanced TTE. In order to overcome this significant limitation, a strict audit diagnosis was necessary. Subsequently, three emergency medical specialists performed the audit diagnosis at least one month after the patient visited the ED, completely blinded to the ultrasound evaluation. Besides, to objectively determine the most crucial diagnosis that caused the patient's chief complaint, discussions were conducted several times through plenary meetings. Second, we performed convenient sampling. All tests were performed by three emergency medical professionals whenever possible during the daytime. Third, there may have been a selection bias in the process, as M-PoCUS was only performed on patients whom the charged emergency physicians requested. Fourth, M-PoCUS performers were not blinded to clinical information, rather they performed the index test after reviewing the chief complaints associated with present illnesses, past medical history, and past radiologic test results. This could bias the diagnostic effectiveness of M-PoCUS. However, we assumed that such way might be helpful to distinguish pathogenic findings in cancer patients, whether acute or chronic. When 
radiologists interpret CT and x-rays, they compare the test result with previous ones. We believe that it should be the same in the PoCUS interpretation used in daily clinical practice for cancer patients in the ED.

\section{Conclusions}

In summary, in this study, M-PoCUS, used collectively with the initial clinical evaluation, was useful to rule out life-threatening conditions in cancer patients who visited the ED during SARS-CoV-2 pandemic. However, PTE was hard to distinguish using only MPoCUS because the patients' characteristics regarding the pre-existing diseases might have been scattered in the thorax. We believe a CT angiography should be encouraged when clinical suspicion is found. A large prospective cohort study is needed to confirm the results of this study, and to determine M-PoCUS' role in terms of clinical efficacy for cancer patients in the ED.

\section{List Of Abbreviations}

ED: emergency department; M-PoCUS: multi-organ point-of-care ultrasonography; PoCUS: Point-of-care ultrasonography; LUS: lung ultrasound; TTE: transthoracic echocardiography; LEVUS: lower extremity vein ultrasound; IVC: inferior vena cava; LV: left ventricle; EF: ejection fraction; RV: right ventricle; RA: right atrium

\section{Declarations}

\section{Ethic approval and consent to participate}

This study was approved by our institutional review board and met the criteria for exemption from informed consent. (Seoul National University IRB No. 2010-110-1165)

\section{Consent for publication}

Not applicable.

\section{Availability of data and materials}

The datasets generated during and/or analyzed during the current study are available from the corresponding author on reasonable request

\section{Competing interests}

The authors declare that they have no competing interests.

\section{Funding}

None

\section{Authors' contributions}

YAC and MSL conceived of the presented idea. YAC, MSL, TKK, and YHL reviewed the ultrasonographic examinations and contributed to the interpretation of the results. KHK, JWP, and SGYL retrospective review all relevant data and made audit diagnoses. YAC and MSL took the lead in writing the manuscript. JYJ and JYK provided critical feedback and helped shape the research, analysis and manuscript. All authors discussed the results and commented on the manuscript. MSL takes responsibility for the paper as a whole.

\section{Acknowledgements}

Not applicable.

\section{References}

1. Jehle D, Davis E, Evans T, Harchelroad F, Martin M, Zaiser K, et al. Emergency department sonography by emergency physicians. The American journal of emergency medicine. 1989;7(6):605-11. 
2. Plummer D. Whose turf is it, anyway? Diagnostic ultrasonography in the emergency department. Academic Emergency Medicine. 2000;7(2):186-7.

3. Abdolrazaghnejad A, Banaie M, Safdari M. Ultrasonography in emergency department; a diagnostic tool for better examination and decision-making. Advanced journal of emergency medicine. 2018;2(1).

4. Barr L, Hatch N, Roque PJ, Wu TS. Basic ultrasound-guided procedures. Critical Care Clinics. 2014;30(2):275-304, vi.

5. Hatch N, Wu TS, Barr L, Roque PJ. Advanced ultrasound procedures. Critical Care Clinics. 2013;30(2):305-29, vi.

6. Alrajhi K, Woo M, Vaillancourt C. Test characteristics of ultrasonography for the detection of pneumothorax: a systematic review and meta-analysis. chest. 2011:11-0131.

7. Alrajab S, Youssef AM, Akkus NI, Caldito G. Pleural ultrasonography versus chest radiography for the diagnosis of pneumothorax: review of the literature and meta-analysis. Critical Care. 2013;17(5):1-8.

8. Hu Q-J, Shen Y-C, Jia L-Q, Guo S-J, Long H-Y, Pang C-S, et al. Diagnostic performance of lung ultrasound in the diagnosis of pneumonia: a bivariate meta-analysis. International journal of clinical and experimental medicine. 2014;7(1):115.

9. Chavez MA, Shams N, Ellington LE, Naithani N, Gilman RH, Steinhoff MC, et al. Lung ultrasound for the diagnosis of pneumonia in adults: a systematic review and meta-analysis. Respiratory research. 2014;15(1):50.

10. Martindale JL, Wakai A, Collins SP, Levy PD, Diercks D, Hiestand BC, et al. Diagnosing acute heart failure in the emergency department: a systematic review and meta-analysis. Academic emergency medicine. 2016;23(3):223-42.

11. Maw AM, Hassanin A, Ho PM, Mclnnes MD, Moss A, Juarez-Colunga E, et al. Diagnostic accuracy of point-of-care lung ultrasonography and chest radiography in adults with symptoms suggestive of acute decompensated heart failure: a systematic review and meta-analysis. JAMA network open. 2019;2(3):e190703-e.

12. Reissig A, Copetti R, Mathis G, Mempel C, Schuler A, Zechner P, et al. Lung ultrasound in the diagnosis and follow-up of community-acquired pneumonia: a prospective, multicenter, diagnostic accuracy study. Chest. 2012;142(4):965-72.

13. Zanobetti M, Poggioni C, Pini R. Can chest ultrasonography replace standard chest radiography for evaluation of acute dyspnea in the ED? Chest. 2011;139(5):1140-7.

14. Nazerian P, Vanni S, Volpicelli G, Gigli C, Zanobetti M, Bartolucci M, et al. Accuracy of point-of-care multiorgan ultrasonography for the diagnosis of pulmonary embolism. Chest. 2014;145(5):950-7.

15. Koenig S, Chandra S, Alaverdian A, Dibello C, Mayo PH, Narasimhan M. Ultrasound assessment of pulmonary embolism in patients receiving CT pulmonary angiography. Chest. 2014;145(4):818-23.

16. Gargani L, Frassi F, Soldati G, Tesorio P, Gheorghiade M, Picano E. Ultrasound lung comets for the differential diagnosis of acute cardiogenic dyspnoea: A comparison with natriuretic peptides $\rrbracket$. European journal of heart failure. 2008;10(1):70-7.

17. Frassi F, Gargani L, Gligorova S, Ciampi Q, Mottola G, Picano E. Clinical and echocardiographic determinants of ultrasound lung comets. European journal of echocardiography. 2007;8(6):474-9.

18. Gargani L. Lung ultrasound: a new tool for the cardiologist. Cardiovascular ultrasound. 2011;9(1):6.

19. Volpicelli G, Lamorte A, Tullio M, Cardinale L, Giraudo M, Stefanone V, et al. Point-of-care multiorgan ultrasonography for the evaluation of undifferentiated hypotension in the emergency department. Intensive care medicine. 2013;39(7):1290-8.

20. Laursen CB, Sloth E, Lambrechtsen J, Lassen AT, Madsen PH, Henriksen DP, et al. Focused sonography of the heart, lungs, and deep veins identifies missed life-threatening conditions in admitted patients with acute respiratory symptoms. Chest. 2013;144(6):1868-75.

21. Laursen CB, Sloth E, Lassen AT, Christensen Rd, Lambrechtsen J, Madsen PH, et al. Point-of-care ultrasonography in patients admitted with respiratory symptoms: a single-blind, randomised controlled trial. The Lancet Respiratory medicine. 2014;2(8):63846.

22. Zanobetti M, Scorpiniti M, Gigli C, Nazerian P, Vanni S, Innocenti F, et al. Point-of-care ultrasonography for evaluation of acute dyspnea in the ED. Chest. 2017;151(6):1295-301.

23. Sadik M, Ozlem K, Huseyin M, AliAyberk B, Ahmet S, Ozgur O. Attributes of cancer patients admitted to the emergency department in one year. World journal of emergency medicine. 2014;5(2):85.

24. Guddati AK, Kumar N, Segon A, Joy PS, Marak CP, Kumar G. Identifying oncological emergencies. Medical Oncology. 2013;30(3):669. 
25. Barbera L, Taylor C, Dudgeon D. Why do patients with cancer visit the emergency department near the end of life? Cmaj. 2010;182(6):563-8.

26. Earle CC, Neville BA, Landrum MB, Ayanian JZ, Block SD, Weeks JC. Trends in the aggressiveness of cancer care near the end of life. Journal of Clinical Oncology. 2004;22(2):315-21.

27. Qureshi D, Tanuseputro P, Perez R, Seow H. Place of care trajectories in the last two weeks of life: a population-based cohort study of Ontario decedents. Journal of palliative medicine. 2018;21(11):1588-95.

28. Vandyk AD, Harrison MB, Macartney G, Ross-White A, Stacey D. Emergency department visits for symptoms experienced by oncology patients: a systematic review. Supportive Care in Cancer. 2012;20(8):1589-99.

29. Kim K, Jung JY, Park J, Lee M, Lee Y. Operating bedside cardiac ultrasound program in emergency medicine residency: a retrospective observation study from the perspective of performance improvement. 2020.

30. Khorana A, Noble S, Lee A, Soff G, Meyer G, O'Connell C, et al. Role of direct oral anticoagulants in the treatment of cancerassociated venous thromboembolism: guidance from the SSC of the ISTH. Journal of Thrombosis and Haemostasis. 2018;16(9):1891-4.

31. Moore CL, Rose GA, Tayal VS, Sullivan DM, Arrowood JA, Kline JA. Determination of left ventricular function by emergency physician echocardiography of hypotensive patients. Academic Emergency Medicine. 2002;9(3):186-93.

32. Vieillard-Baron A, Charron C, Chergui K, Peyrouset O, Jardin F. Bedside echocardiographic evaluation of hemodynamics in sepsis: is a qualitative evaluation sufficient? Intensive care medicine. 2006;32(10):1547-52.

33. Committee S. Single-bolus tenecteplase plus heparin compared with heparin alone for normotensive patients with acute pulmonary embolism who have evidence of right ventricular dysfunction and myocardial injury: rationale and design of the Pulmonary Embolism Thrombolysis (PEITHO) trial. American heart journal. 2012;163(1):33-8. e1.

34. Jaff MR, McMurtry MS, Archer SL, Cushman M, Goldenberg N, Goldhaber SZ, et al. Management of massive and submassive pulmonary embolism, iliofemoral deep vein thrombosis, and chronic thromboembolic pulmonary hypertension: a scientific statement from the American Heart Association. Circulation. 2011;123(16):1788-830.

35. Rudski LG, Lai WW, Afilalo J, Hua L, Handschumacher MD, Chandrasekaran K, et al. Guidelines for the echocardiographic assessment of the right heart in adults: a report from the American Society of Echocardiography: endorsed by the European Association of Echocardiography, a registered branch of the European Society of Cardiology, and the Canadian Society of Echocardiography. Journal of the American Society of Echocardiography. 2010;23(7):685-713.

36. Reydel B, Spodick DH. Frequency and significance of chamber collapses during cardiac tamponade. The American heart journal. 1990;119(5):1160-3.

37. Zhang S, Kerins DM, III BFB. Doppler echocardiography in cardiac tamponade and constrictive pericarditis. Echocardiography. 1994;11(5):507-21.

38. Lang RM, Bierig M, Devereux RB, Flachskampf FA, Foster E, Pellikka PA, et al. Recommendations for chamber quantification: a report from the American Society of Echocardiography's Guidelines and Standards Committee and the Chamber Quantification Writing Group, developed in conjunction with the European Association of Echocardiography, a branch of the European Society of Cardiology. Journal of the American Society of Echocardiography. 2005;18(12):1440-63.

39. Volpicelli G, Elbarbary M, Blaivas M, Lichtenstein DA, Mathis G, Kirkpatrick AW, et al. International evidence-based recommendations for point-of-care lung ultrasound. Intensive care medicine. 2012;38(4):577-91.

40. Volpicelli G, Mussa A, Garofalo G, Cardinale L, Casoli G, Perotto F, et al. Bedside lung ultrasound in the assessment of alveolarinterstitial syndrome. The American journal of emergency medicine. 2006;24(6):689-96.

41. Ha Y-R, Toh H-C. Clinically integrated multi-organ point-of-care ultrasound for undifferentiated respiratory difficulty, chest pain, or shock: a critical analytic review. Journal of Intensive Care. 2016;4(1):1-19.

42. Falcetta A, Leccardi S, Testa E, Papaleo F, Fenoglio L, Melchio R. The role of lung ultrasound in the diagnosis of interstitial lung. 2018.

43. Volpicelli G, Caramello V, Cardinale L, Mussa A, Bar F, Frascisco MF. Detection of sonographic B-lines in patients with normal lung or radiographic alveolar consolidation. Medical Science Monitor. 2008;14(3):CR122-CR8.

44. Lichtenstein DA. Lung ultrasound in the critically ill. Annals of intensive care. 2014;4(1):1.

45. Yang P-C, Luh K-T, Chang D-B, Wu H-D, Yu C, Kuo S. Value of sonography in determining the nature of pleural effusion: analysis of 320 cases. AJR American journal of roentgenology. 1992;159(1):29-33.

Page 9/16 
46. Lomas D, Padley S, Flower C. The sonographic appearances of pleural fluid. The British journal of radiology. 1993;66(787):61924.

47. Brockelsby C, Ahmed M, Gautam M. P1 Pleural effusion size estimation: US, CXR or CT? : BMJ Publishing Group Ltd; 2016.

48. Razazi K, Thille AW, Carteaux G, Beji O, Brun-Buisson C, Brochard L, et al. Effects of pleural effusion drainage on oxygenation, respiratory mechanics, and hemodynamics in mechanically ventilated patients. Annals of the American Thoracic Society. 2014;11(7):1018-24.

49. Balik M, Plasil P, Waldauf P, Pazout J, Fric M, Otahal M, et al. Ultrasound estimation of volume of pleural fluid in mechanically ventilated patients. Intensive care medicine. 2006;32(2):318.

50. Crisp JG, Lovato LM, Jang TB. Compression ultrasonography of the lower extremity with portable vascular ultrasonography can accurately detect deep venous thrombosis in the emergency department. Annals of emergency medicine. 2010;56(6):601-10.

51. Landis JR, Koch GG. The measurement of observer agreement for categorical data. biometrics. 1977:159-74.

52. Squizzato A, Rancan E, Dentali F, Bonzini M, Guasti L, Steidl L, et al. Diagnostic accuracy of lung ultrasound for pulmonary embolism: a systematic review and meta-analysis. Journal of thrombosis and haemostasis. 2013;11(7):1269-78.

\section{Tables}




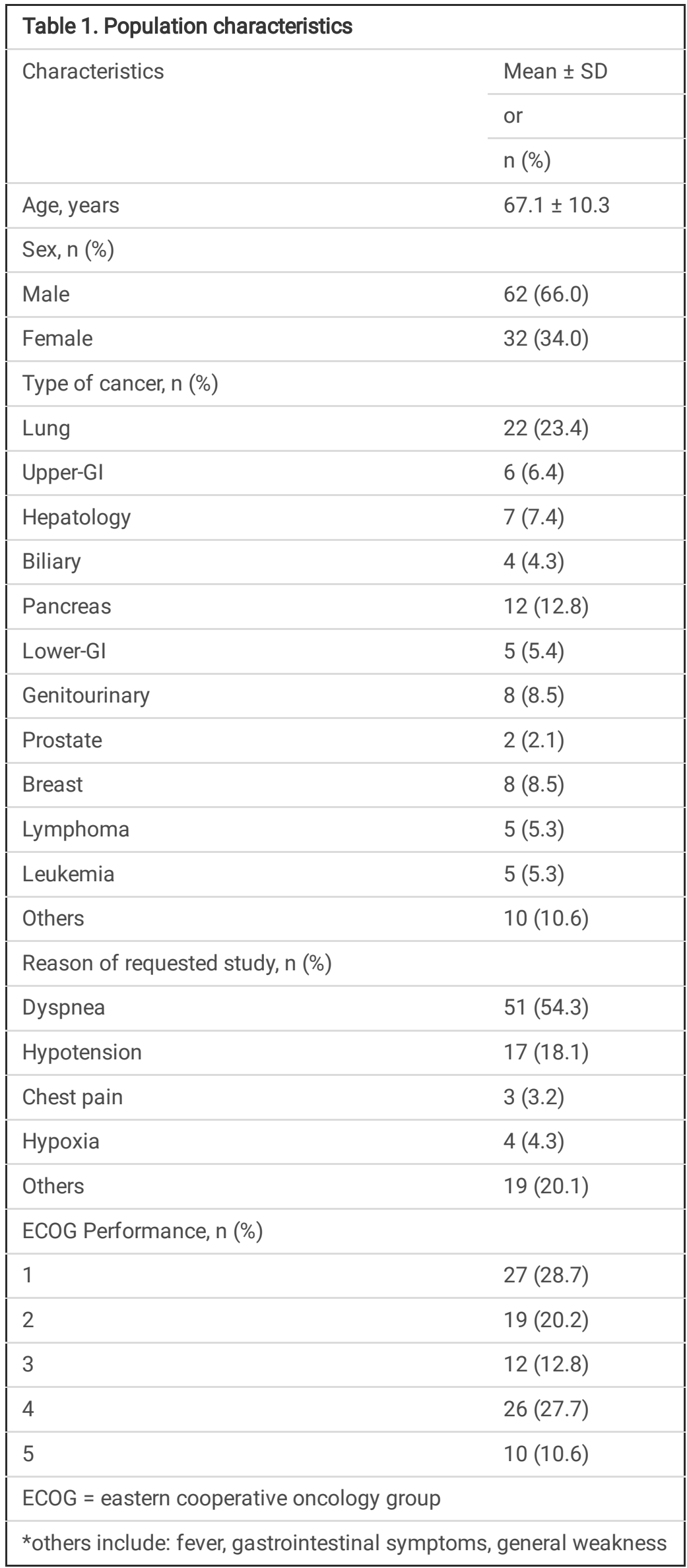




\begin{tabular}{|lcc|}
\hline \multicolumn{3}{|l|}{ Table 2. Time (minute) to intervention from arrival at ED } \\
\hline Characteristics & $\mathrm{n}$ & Median (IQR) \\
\hline M-PoCUS evaluation time* & 94 & $10(7.0-14.0)$ \\
\hline M-PoCUS & 94 & $63(39.0-195.0)$ \\
\hline Chest x-ray & 88 & $75(46.0-111.5)$ \\
\hline Computed tomography & 62 & $240.5(148.0-467.0)$ \\
\hline Antibiotic & 53 & $138(70.0-241.0)$ \\
\hline Fluid loading & 39 & $88(50.5-122.5)$ \\
\hline Intravenous diuretic & 9 & $85(53.0-135.0)$ \\
\hline Pleurocentesis & 9 & $156(71.0-677.0)$ \\
\hline $\begin{array}{l}\text { Pericardiocentesis } \\
\text { M-PoCUS = multi-organ point-of-care ultrasound; IQR = interquartile range } \\
\text { *M-PoCUS evaluation time is spending time to complete M-PoCUS examination. }\end{array}$ \\
\hline
\end{tabular}




\begin{tabular}{|c|c|}
\hline \multicolumn{2}{|l|}{ Table 3. M-PoCUS Findings } \\
\hline Findings & Value $(n=94)$ \\
\hline \multicolumn{2}{|l|}{ Heart, No. (\%) } \\
\hline Hyperdynamic LV function & $49(52.1)$ \\
\hline Normal LV function & $36(38.4)$ \\
\hline Reduced LV function & $5(5.3)$ \\
\hline Severe depressed LV function & $2(2.1)$ \\
\hline No access visual systolic function & $2(2.1)$ \\
\hline Pericardial effusion without tamponade feature & $10(10.6)$ \\
\hline Pericardial effusion with tamponade feature & $3(3.2)$ \\
\hline Marked dilatation of the Right ventricle & $2(2.1)$ \\
\hline \multicolumn{2}{|l|}{ Inferior vena cava, No. (\%) } \\
\hline Normal & $20(21.3)$ \\
\hline IVC with signs of intravascular volume depletion & $47(50.0)$ \\
\hline IVC with signs of markedly increased right atrium pressure & $10(10.6)$ \\
\hline Not possible to or no assess IVC using sonography & $17(18.1)$ \\
\hline \multicolumn{2}{|l|}{ Lung, No. (\%) } \\
\hline Normal & $39(41.5)$ \\
\hline Pleural abnormality & $11(11.7)$ \\
\hline Pneumothorax & $0(0.0)$ \\
\hline Focal interstitial syndrome & $19(20.2)$ \\
\hline Diffuse interstitial syndrome & $19(20.2)$ \\
\hline Unilateral consolidation & $26(27.7)$ \\
\hline Bilateral consolidation & $36(38.3)$ \\
\hline Simple pleural effusion & $30(31.9)$ \\
\hline Complex pleural effusion & $10(10.6)$ \\
\hline \multicolumn{2}{|l|}{ Lower extremity, No. (\%) } \\
\hline No signs of DVT & $16(17.0)$ \\
\hline Signs of DVT & $1(1.1)$ \\
\hline No access lower extremity vein & $77(81.9)$ \\
\hline
\end{tabular}




\begin{tabular}{|c|c|c|c|c|c|c|c|c|c|c|}
\hline & CPE & $\begin{array}{l}\text { Bilateral } \\
\text { AlS }\end{array}$ & $\begin{array}{l}\text { Unilateral } \\
\text { AIS }\end{array}$ & $\begin{array}{l}\text { Pleural } \\
\text { effusion }\end{array}$ & PTE & Tamponade & $\begin{array}{l}\text { Hypovolemic/ } \\
\text { Distributive }\end{array}$ & PTX & Normal & $\begin{array}{l}\text { Tumor } \\
\text { progression }\end{array}$ \\
\hline $\begin{array}{l}\text { M- } \\
\text { PoCUS } \\
\text { No. } \\
\text { (\%) }\end{array}$ & $\begin{array}{l}3 \\
(3.2)\end{array}$ & $\begin{array}{l}25 \\
(26.6)\end{array}$ & $\begin{array}{l}11 \\
(11.7)\end{array}$ & $\begin{array}{l}16 \\
(17.0)\end{array}$ & $\begin{array}{l}0 \\
(0.0)\end{array}$ & $\begin{array}{l}3 \\
(3.2)\end{array}$ & $\begin{array}{l}29 \\
(30.9)\end{array}$ & $\begin{array}{l}0 \\
(0.0)\end{array}$ & $\begin{array}{l}7 \\
(7.4)\end{array}$ & $\begin{array}{l}23 \\
(24.5)\end{array}$ \\
\hline $\begin{array}{l}\text { Audit } \\
\text { No. } \\
(\%)\end{array}$ & $\begin{array}{l}3 \\
(3.2)\end{array}$ & $\begin{array}{l}26 \\
(27.7)\end{array}$ & $\begin{array}{l}7 \\
(7.4)\end{array}$ & $\begin{array}{l}13 \\
(13.8)\end{array}$ & $\begin{array}{l}4 \\
(4.3)\end{array}$ & $\begin{array}{l}3 \\
(3.2)\end{array}$ & $\begin{array}{l}31 \\
(33.0)\end{array}$ & $\begin{array}{l}0 \\
(0.0\end{array}$ & $\begin{array}{l}7 \\
(7.4)\end{array}$ & $\begin{array}{l}26 \\
(27.7)\end{array}$ \\
\hline \multicolumn{11}{|c|}{$\begin{array}{l}\mathrm{CPE}=\text { cardiogenic pulmonary edema; } \mathrm{AIS}=\text { alveolar interstitial syndrome; } \mathrm{PTE}=\text { pulmonary thromboembolism; } \mathrm{PTX}= \\
\text { pneumothorax; Normal = normal or other }\end{array}$} \\
\hline
\end{tabular}

\begin{tabular}{|c|c|c|c|}
\hline \multirow[b]{2}{*}{ Test } & \multicolumn{3}{|c|}{ Life-threatening condition } \\
\hline & Present & Absent & Total \\
\hline M-Pocus positive & 19 & 3 & 22 \\
\hline M-Pocus negative & 4 & 68 & 72 \\
\hline Total & 23 & 71 & 94 \\
\hline
\end{tabular}




\section{Table 6. Definite diagnosis}

Diagnosis

Value $(n=118)$

Heart, No. (\%)

Tamponade

Cardiogenic pulmonary edema

Lung, No. (\%)

Pneumonia*

29 (30.9)

Pleural effusion

$15(16.0)$

Hypovolemia, No. (\%)

Hemorrhage, symptomatic anemia

Dehydration, hypoalbuminemia, prerenal AKI, DKA, ileus

$12(12.8)$

Septic shock, No. (\%)

GI origin

$8(8.5)$

Genitourinary origin

$1(1.1)$

Necrotizing fasciitis

Inconclusive origin

$1(1.1)$

Venous thromboembolism, No. (\%)

Pulmonary embolism

Pulmonary embolism + deep vein thrombosis

Increased tumor burden, No. (\%)

Radiologically confirmed

Clinically suspected

$13(13.8)$

Others, No. (\%)

Postrenal AKI

Neutropenic fever

Cellulitis

Normal

$\mathrm{AKI}$ = acute kidney injury; $\mathrm{GI}$ = gastrointestinal; DKA = diabetic ketoacidosis;

Total $\mathrm{n}=118$, two concomitant diagnoses could be present

*Pneumonia category includes not only infectious pneumonia, but also drug induced pneumonitis and aspiration pneumonitis.

Patients with pneumonia septic shock were categorized under pneumonia. 


\section{Figures}

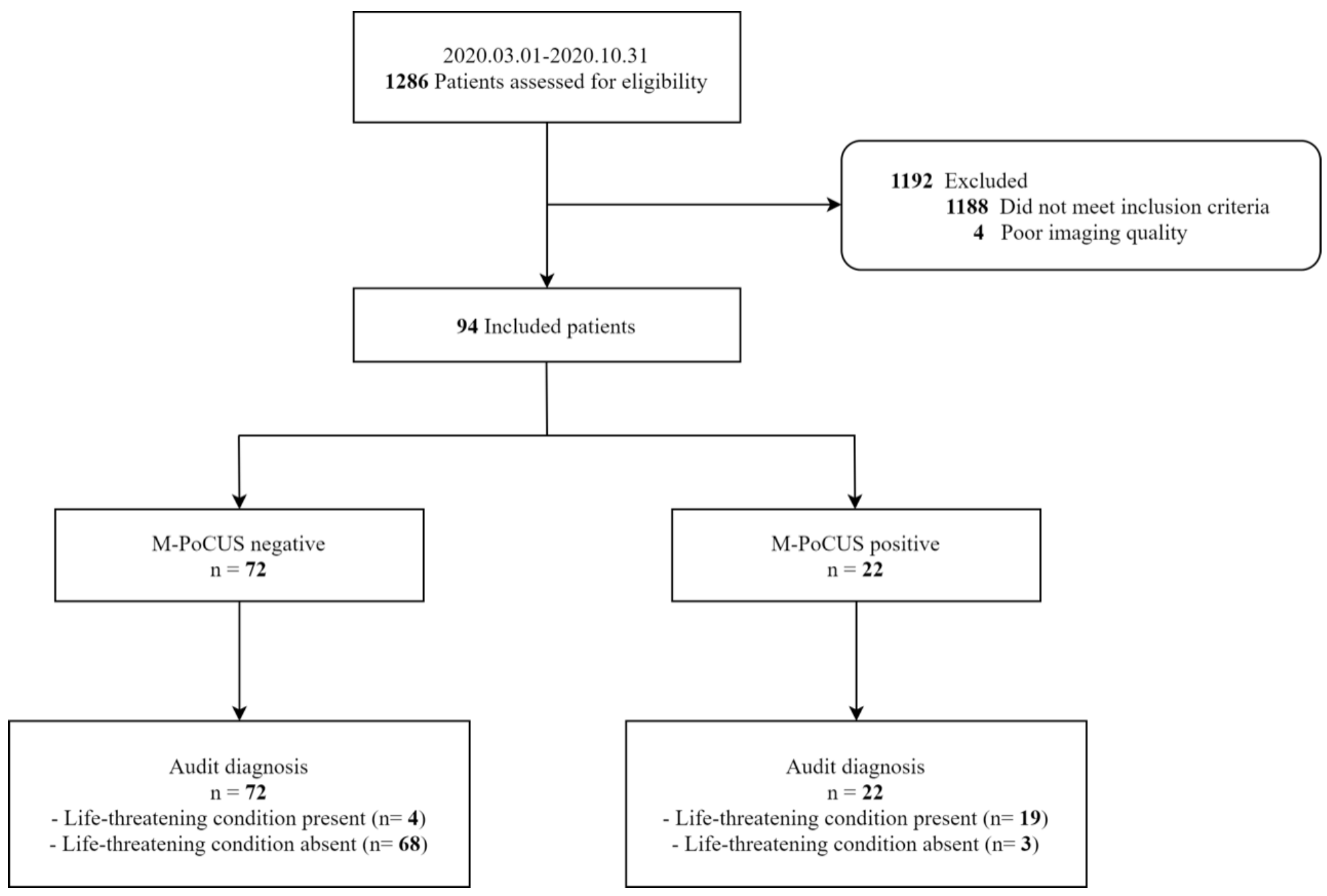

\section{Figure 1}

Participants flow chart, M-PoCUS = multi-organ point-of-care ultrasound

\section{Supplementary Files}

This is a list of supplementary files associated with this preprint. Click to download.

- Additionalfile1.pdf

- Additionalfile2.pdf 